This is an electronic reprint of the original article. This reprint may differ from the original in pagination and typographic detail.

Author(s): Pavlyukh, Y.; Uimonen, A.-M.; Stefanucci, G.; van Leeuwen, Robert

Title: Vertex Corrections for Positive-Definite Spectral Functions of Simple Metals

Year: $\quad 2016$

Version:

Please cite the original version:

Pavlyukh, Y., Uimonen, A.-M., Stefanucci, G., \& van Leeuwen, R. (2016). Vertex Corrections for Positive-Definite Spectral Functions of Simple Metals. Physical Review Letters, 117(20), Article 206402. https://doi.org/10.1103/PhysRevLett.117.206402

All material supplied via JYX is protected by copyright and other intellectual property rights, and duplication or sale of all or part of any of the repository collections is not permitted, except that material may be duplicated by you for your research use or educational purposes in electronic or print form. You must obtain permission for any other use. Electronic or print copies may not be offered, whether for sale or otherwise to anyone who is not an authorised user. 


\title{
Vertex Corrections for Positive-Definite Spectral Functions of Simple Metals
}

\author{
Y. Pavlyukh* \\ Institut für Physik, Martin-Luther-Universität Halle-Wittenberg, 06120 Halle, \\ Germany and Department of Physics and Research Center OPTIMAS, University of Kaiserslautern, \\ P.O. Box 3049, 67653 Kaiserslautern, Germany
}

A.-M. Uimonen

Clarendon Laboratory, Department of Physics, University of Oxford, Parks Road, Oxford OX1 3PU, United Kingdom

G. Stefanucci

Dipartimento di Fisica and European Theoretical Spectroscopy Facility (ETSF),

Università di Roma Tor Vergata, Via della Ricerca Scientifica 1, 00133 Rome,

Italy and INFN, Sezione di Roma Tor Vergata, Via della Ricerca Scientifica 1, 00133 Roma, Italy

R. van Leeuwen

Department of Physics and European Theoretical Spectroscopy Facility (ETSF), Nanoscience Center, University of Jyväskylä, FI-40014 Jyväskylä, Finland

(Received 14 July 2016; published 10 November 2016)

\begin{abstract}
We present a systematic study of vertex corrections in a homogeneous electron gas at metallic densities. The vertex diagrams are built using a recently proposed positive-definite diagrammatic expansion for the spectral function. The vertex function not only provides corrections to the well known plasmon and particle-hole scatterings, but also gives rise to new physical processes such as the generation of two plasmon excitations or the decay of the one-particle state into a two-particle-one-hole state. By an efficient Monte Carlo momentum integration we are able to show that the additional scattering channels are responsible for a reduction of the bandwidth, the appearance of a secondary plasmon satellite below the Fermi level, and a substantial redistribution of spectral weights. The feasibility of the approach for first-principles band-structure calculations is also discussed.
\end{abstract}

DOI: 10.1103/PhysRevLett.117.206402

Starting from the introduction of the notion of the quasiparticle (QP) [1] as an elemental excitation in Fermi liquids [2] we have almost a complete picture of its on-shell properties [3,4]. Quantum Monte Carlo (QMC) simulations [5,6], phase diagrams [7-12], structure factors [13], and effective interparticle interactions [14] contributed to our knowledge of Fermi liquids and to the development of the density functionals $[15,16]$.

Despite these achievements we still have a poor knowledge of the energy- and momentum-resolved spectral function $A(k, \omega)$ away from the on-shell manifold, i. e., when $\omega \not \varepsilon_{k}$. In angular resolved photoemission this is the regime where electrons with reduced energy (as compared to the prediction based on band structure and energy balance) are observed. Self-consistent (SC) perturbation theory, e.g., SC GW [17], accurately predicts total energies [18-20] and it is fully conserving at the one-particle level. The latter is a crucial property in the description of transport phenomena [21]. However, for spectral properties SC schemes do not show the expected improvement over simpler one-shot calculations [22-24]. In fact, they suffer from serious drawbacks: the incoherent background in the spectral function gains weight at the expense of the QP peak [25], the bandwidth is largely overestimated [26,27], and the screened interaction does not obey the $f$-sum rule $[28,29]$. It was then proposed that self-energy (SE) diagrams with vertex corrections may cancel the spurious SC effects [25,30-33]. This fueled a number of notable attempts to include the vertex function in a model fashion: using the plasmon model for the screened interaction [34], neglecting the incoherent part of the electron spectral function [35], and employing the Ward identities and a model form of the exchange-correlation kernel [27,36-38], or the cumulant expansions [39-45]. Although these methods clarified a number of issues, they do not provide an exhaustive picture [46]. The major obstacle for a full-fledged vertex calculation, besides numerical complexity, is the issue of negative spectral densities, first noted by Minnhagen [34,50] and only recently solved by us using a positive-definite diagrammatic expansion [51,52]. Our solution merges many-body perturbation theory and scattering theory, thus returning a positive-semidefinite (PSD) spectral function by construction.

With the PSD tool at our disposal, in this Letter we investigate the influence of vertex corrections on the spectral function of the homogeneous electron gas (HEG) and demonstrate that it leads to a number of novel physical phenomena that cannot be reduced to mere self-consistency cancellations. 
Let us motivate and discuss the PSD approximation used in this work. In terms of dressed electronic propagators and screened interaction $W$ there is a single second order SE diagram $\Sigma^{(2)}={ }^{2}$ however, yields negative spectra in some frequency regions. This prohibits the usual probability interpretation and, even worse, it jeopardizes SC calculations since the resulting Green's function (GF) has the wrong analytic structure [53]. The key idea of the PSD scheme [51,52] consists in (1) writing a SE diagram as the sum of its partitions, i.e., diagrams with particle and hole propagators, (2) bisecting each partition into two half diagrams, (3) adding the missing half diagrams to form a perfect square, and (4) gluing the half diagrams back. For $\Sigma^{(2)}$ the half diagrams, see Figs. 1(a)-1(c), contain scatterings with up to three particles and two holes in the final state [51]. The SE partitions stem from the interference between these scatterings and after the PSD treatment one obtains partitions up to the fourth order in $W$, see Ref. [51] for the full list. Among them there are three that deserve special attention. $\Sigma_{a a}$ in Fig. 1(d) results from the interference of scattering $(a)$ with itself. As illustrated in Fig. $1(\mathrm{~g}) \Sigma_{a a}$ involves an electron-hole $(e-h)$ pair (orange area) or a plasmon in the final state (this is the first order effect described by the $G W \mathrm{SE}$ ). The plus and minus vertices in the SE partitions have the purpose of distinguishing the constituent half diagrams (resulting from the cut of all propagators with $+/-$ and $-/+$ vertices). $\Sigma_{a \bar{a}}$ in Fig. 1(e) is formed by the interference between the scattering $(a)$, leading to a two-particle-one-hole $\left(p_{f_{1}}-p_{f_{2}}-q_{f}\right)$ final state, and the same scattering with interchanged particle momenta (indicated with $\bar{a}$ ), see Fig. 1(h). Finally, $\Sigma_{c \bar{c}}$ in Fig. 1(f) is formed by the interference between the scattering $(c)$, in which a particle loses its energy by exciting two $e-h$ pairs, two plasmons, or a (a)

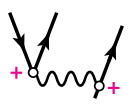

(d)

(g)

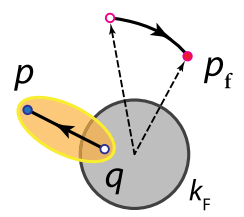

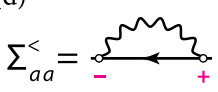

(b)

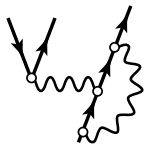

(e)

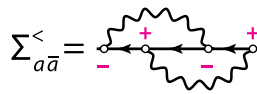

(h)

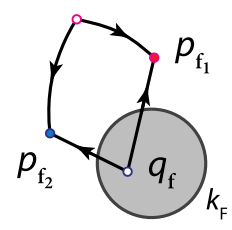

(c)

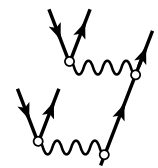

(f)

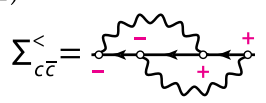

(i)

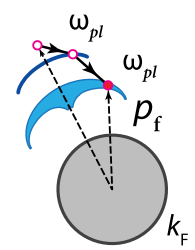

FIG. 1. (a)-(c) The half diagrams emerging from the bisection of the $\Sigma^{(2)}$ partitions (the wiggly lines denote the screened interaction). (d)-(f) Three partitions of the PSD self-energy and (g)-(i) their momentum space representation. mixture of them, and the same scattering with interchanged particle and hole momenta, see Fig. 1(i). Plasmon generation is a dominant second order scattering process although it has a severely limited phase space (dark blue line and light blue area) due to energy and momentum conservation. Higher order terms in $W$ (up to fourth order) arise from other interferences and are needed to ensure the overall positivity [51]. In general the PSD procedure leads to a manifestly positive Fermi's "golden rule" form of the SE, $\Sigma^{<}(k, \omega) \sim$ $\sum_{n, f} \Gamma^{(n)}(k, \omega)\left|1+r_{s} \gamma_{1}^{(n)}+r_{s}^{2} \gamma_{2}^{(n)}+\cdots\right|^{2} \delta\left(\omega+\epsilon_{k}-E_{f}^{(n)}\right)$, where the sum runs over all final states of energy $E_{f}^{(n)}$ with $(n+1)$ particles and $n$ holes ( $r_{s}$ being the Wigner-Seitz radius). The role of high order diagrams is twofold: they bring new scattering mechanisms into play (hence new rates $\Gamma^{(n)}$ ) and renormalize them through the perturbative corrections $\gamma_{i}^{(n)}$.

We already mentioned that one of the motivations for including diagrams beyond $G W$ is the excessive broadening of the spectral features when the level of selfconsistency is increased, e.g., $G^{(0)} W^{(0)} \rightarrow G W^{(0)} \rightarrow G W$. As a full SC calculation of the PSD SE of Ref. [51] is out of reach, we partially account for self-consistency by using a $G^{(0)} W^{(0)}$ GF (finite QP broadening and plasmon satellites) and a RPA screened interaction. Our calculations indicate that higher-order diagrams (aside from bringing in new spectral features) counteract the undesired SC effects, thus suggesting the occurrence of sizable cancellations. We then explore the possibility of producing the PSD results with fewer diagrams and bare GFs. In the bare GF the chemical potential $\mu$ is iteratively adjusted by imposing that the energy of states on the Fermi sphere (where the discontinuity in the momentum distribution $n_{k}$ occurs) is exactly equal to $\mu$ [54]. In Fig. 2(a) we compare the rate
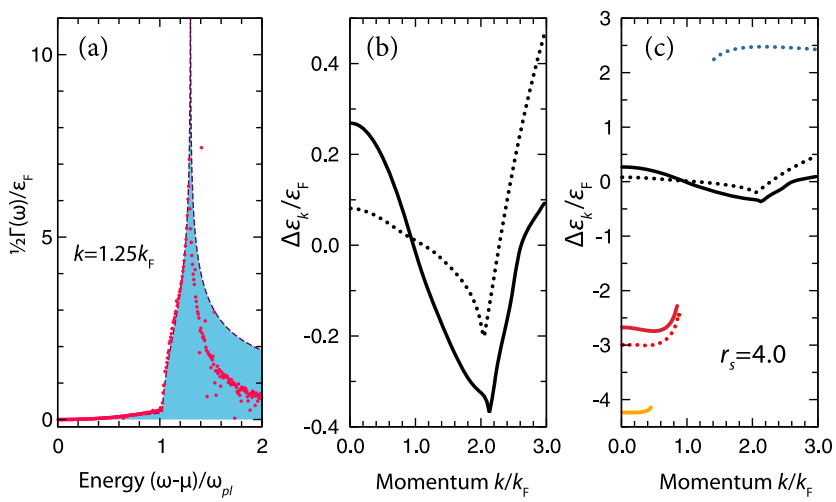

FIG. 2. (a) Rate $\Gamma(k, \omega)$ for $k=1.25 k_{F}$ calculated from the PSD SE of Ref. [51] with the $G^{(0)} W^{(0)}$ GF (red dots) and from the SE $\Sigma=\Sigma_{a \bar{a}}+\Sigma_{c \bar{c}}+\Sigma_{a a}$ with the QP GF (dashed). (b) QP energy correction $\Delta \epsilon_{k}=\epsilon_{k}-\epsilon_{k}^{(0)}$ and (c) dispersion of the plasmon satellites for $G^{(0)} W^{(0)}$ (dotted) and our vertex approximation (solid). The corrections to $\mu$ (with respect to the mean-field value) are $\Delta \mu=-1.76 \epsilon_{F}$ and $\Delta \mu=-1.81 \epsilon_{F}$, respectively. 


$$
\Gamma(k, \omega) \equiv i\left[\Sigma^{R}(k, \omega)-\Sigma^{A}(k, \omega)\right],
$$

as obtained from the PSD diagrams of Ref. [51] with the $G^{(0)} W^{(0)}$ GF and from the much simpler $\Sigma=\Sigma_{a \bar{a}}+\Sigma_{c \bar{c}}+$ $\Sigma_{a a}$ with the QP GF (in both cases we used a RPA $W$ ). The left flank and the hight of the peak are in perfect agreement. At energies in the region of plasmon satellites the full PSD rate decays faster but the trend is similar and the impact of this discrepancy on the spectral function is only minor. More calculations at different $k$ (not shown) confirm the agreement between the two SEs. We therefore infer that the relevant scattering mechanisms for a positive-conserving, leading-order vertex correction are those of Figs. 1(g)-1(i). This reduction of diagrams represents an important advance in view of the correlated band-structure calculations of solids. In the following we use the vertex correction of Figs. 1(d)-1(f) to calculate the QP and plasmon energy dispersions, spectral function, scattering rate, renormalization factor, and momentum distribution.

Results.-The electron density and the dimensionality completely determine the properties of the HEG; in the 3D case they fix the Fermi momentum and energy as follows: $k_{F}=\left(\alpha r_{s}\right)^{-1}$ and $\epsilon_{F}=1 / 2\left(\alpha r_{s}\right)^{-2}$ with $\alpha=[4 /(9 \pi)]^{1 / 3}$. We consider the case of metallic densities $r_{s}=4.0 a_{B}$ appropriate for, e. g., bulk Na metal. Angle-resolved photoemission experiments have pointed out a substantial narrowing of the occupied band in sodium [55] (bandwidth smaller by $20 \%$ as compared to the noninteracting electron dispersion). Although the spectral function of the HEG is not directly comparable with angle-resolved photoemission experiments, estimating the impact of vertex corrections is crucial to quantify extrinsic and surface effects [26,27,56,57]. Our diagrammatic approximation for the vertex provides a bandwidth reduction of $27.5 \%$ (as compared to the noninteracting case), see Fig. 2(b) and the Supplemental Material [58] for the analysis of Na. Figure 2(c) also shows the dispersion of plasmon satellites (red and blue curves).

In the calculations with vertex corrections (solid) the high-energy plasmon branch smears out and, unlike in the $G^{(0)} W^{(0)}$ approximation (dotted), there is no real solution to the Dyson equation. The low energy plasmon is only slightly affected by the vertex. At small momenta the $G^{(0)} W^{(0)}$ position of the plasmon satellite [which is $50 \%$ off the value of $\omega_{\mathrm{PL}}=1.881 \epsilon_{F}$ ] is improved to a small degree. For momenta close to $k_{F}$ the distance from the QP energy reduces but it remains larger than $\omega_{\mathrm{PL}}$. This agrees with the fact that close to the Fermi energy the plasmon satellite is shifted downward by the QP-plasmon interaction [68]. Of note, the vertex is also responsible for the emergence of a second branch at a lower energy (orange) whose position is only $12 \%$ off $2 \omega_{\mathrm{PL}}$. Higher order diagrams are necessary to describe the higher order plasmon replica and to improve the position of the low-energy plasmon satellites [58].

Vertex corrections have a sizable impact on the energy and momentum resolved spectral function

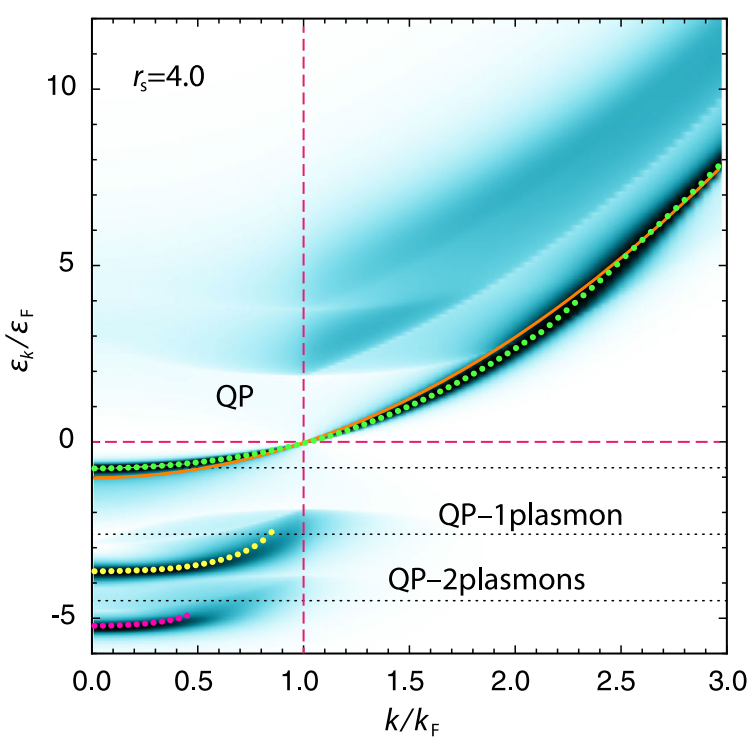

FIG. 3. Energy- and momentum-resolved spectral function with vertex corrections. The solid lines denote the free electron dispersion. The dots denote the solutions of the real Dyson equation, see the main text. For some momentum values multiple solutions (marked with different colors) are obtained.

$A(k, \omega)=i\left[G^{R}-G^{A}\right](k, \omega)$. In Fig. 3 we display the color plot of $A(k, \omega)$ computed with our method. The dotted lines denote the solutions of the real part of the Dyson equation (used to produce the curves in Fig. 2): $\omega+\Delta \mu-\epsilon_{k}^{(0)}=$ $\operatorname{Re}\left[\Sigma^{R}(k, \omega)\right]$. Compared to $G^{(0)} W^{(0)}$ [24] the appearance of the second plasmon satellite below $\mu$, the redistribution of the spectral weight between the first and second satellite, and the broadening of plasmonic spectral features above $\mu$ are the most important findings of this work. They confirm the plasmon-pole model analysis of Ref. [35] that predicted only hole satellites and much broader particle features.

It is interesting to notice that vertex corrections make the QP peak sharper. This can be inferred from the explicit SE expression or from the rate $\Gamma$ of Eq. (1), which we plot in Figs. 4(a)-4(c) for three different values of the momentum. Plasmons do not contribute to the on-shell properties at energies around $\mu$ because they carry finite energy at zero momentum. Instead, the lifetime of the QPs in the vicinity of the Fermi sphere is mainly determined by $\Sigma_{a a}(G W \mathrm{SE})$ involving $e-h$ production or by $\Sigma_{a \bar{a}}(Q P \rightarrow 3 Q P)$. The latter, shown as a yellow shaded curve in Figs. 4(a)-4(c), contributes with a negative sign and leads to the observed reduction of $\Gamma$ (hence to an enhancement of the QP peak) [69]. Such a behavior (alternating series in $\alpha r_{s}$ ) is typical of many perturbation theories. Notice that $\Sigma_{a a}+\Sigma_{a \bar{a}}$ also dominates the asymptotic $(\omega \rightarrow \infty)$ behavior. The scattering with the generation of two plasmons, contained in $\Sigma_{c \bar{c}}$, plays a crucial role for the off-shell properties as it gives rise to new spectral peaks, see the green shaded curve.

In the vicinity of a QP or plasmon (PL) peak the spectral function acquires the form [73] 

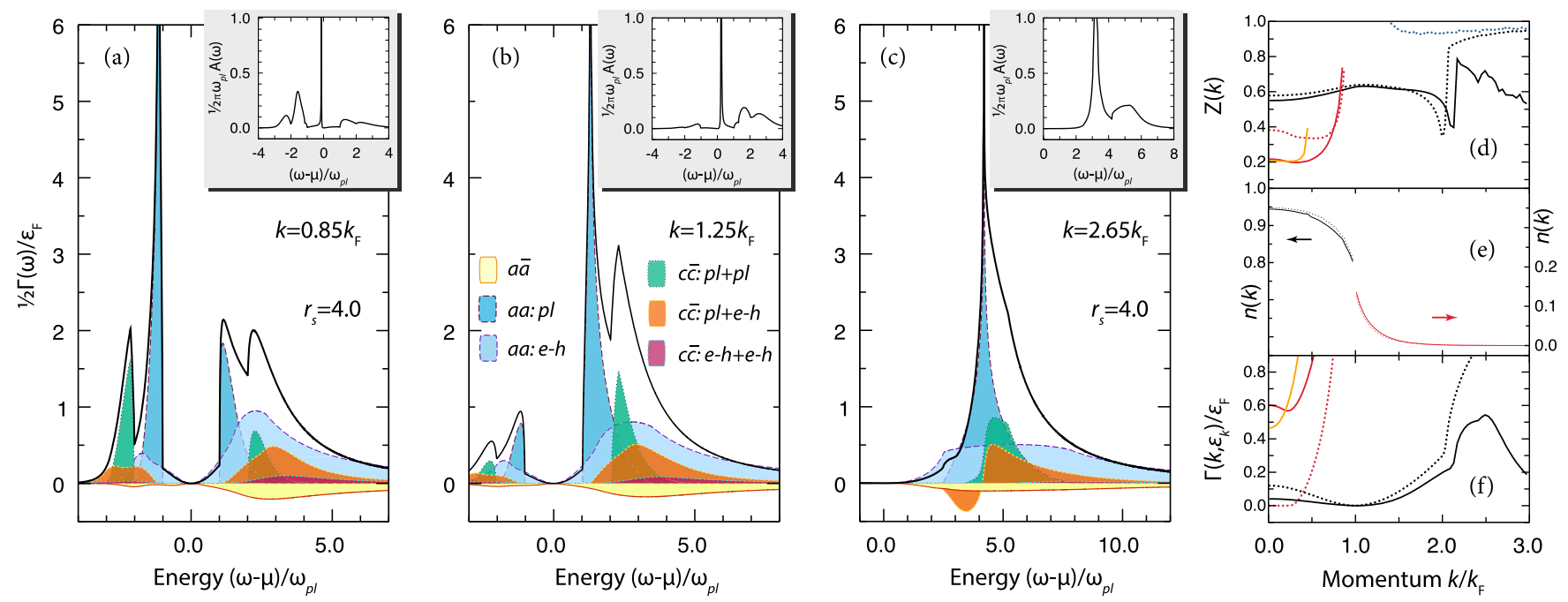

FIG. 4. (a)-(c) $\Gamma(k, \omega)$ for three momentum values. The black line is the total contribution from the SE diagrams $a \bar{a}, c \bar{c}$, and $a a$. The contribution of each diagram is separated according to the intermediate states ( $e-h$ states or plasmons) involved, and it is displayed in different colors. Electron spectral functions are shown in the insets. (d) Renormalization factor of the QP and PL excitations. (e) Momentum occupation number $n_{k}$. (f) Broadening of the QP and PL excitations. For panels (d)-(f) we show the results using $G^{(0)} W^{(0)}$ (dotted) and our vertex approximation (solid).

$$
A(k, \omega)=Z^{(\alpha)}(k) \frac{1 / \tau_{k}^{(\alpha)}}{\left(\omega-\epsilon_{k}^{(\alpha)}\right)^{2}+1 /\left(2 \tau_{k}^{(\alpha)}\right)^{2}},
$$

where $\alpha=\mathrm{QP}$, PL and $\epsilon_{k}^{(\mathrm{QP})}=\epsilon_{k}$, whereas $\epsilon_{k}^{(\mathrm{PL})}$ is the dispersion of the plasmon satellites. This expression contains two quantities of physical interest that we computed using our vertex function: the renormalization factor

$$
Z^{(\alpha)}(k)=\left(1-\left.\frac{\partial}{\partial \omega} \operatorname{Re} \Sigma^{R}(k, \omega)\right|_{\omega=\epsilon_{k}^{(\alpha)}}\right)^{-1},
$$

and the broadening of the QP or PL excitations $1 / \tau_{k}^{(\alpha)}=Z^{(\alpha)}(k) \Gamma\left(k, \epsilon_{k}^{(\alpha)}\right)$.

The renormalization factor is shown in Fig. 4(d). At the band bottom $(k=0) G^{(0)} W^{(0)}$ gives only one plasmon satellite $Z^{(\mathrm{PL})}=0.382$ whereas our vertex approximation gives two satellites with comparable weight $Z^{(\mathrm{PL})}=0.217$ and $Z^{(2 \mathrm{PL})}=0.207$. Furthermore, the QP weight is reduced from $Z^{(\mathrm{QP})}=0.578$ (in $G^{(0)} W^{(0)}$ ) to $Z^{(\mathrm{QP})}=0.550$, indicating that the incoherent part of the spectrum gains weight. These two effects cannot be seen in the cumulant expansion scheme using a $G^{(0)} W^{(0)}$ self-energy input [40], which suppresses $Z$ of the higher plasmon satellites according to the Poissonian distribution [74] and, due to the neglect of the coupling between particle and hole seas, yields the same $Z^{(\mathrm{QP})}$ as in $G^{(0)} W^{(0)}$ [40].

For $k=k_{F}$ the vertex corrections reduce only slightly the $G^{(0)} W^{(0)}$ QP renormalization factor. It is known that SC $G W$ overestimates $\left(Z^{(\mathrm{QP})}=0.793\right)$ the already good $G^{(0)} W^{(0)}$ value $Z^{(\mathrm{QP})}=0.638$ (our calculation) or $Z^{(\mathrm{QP})}=$ 0.646 (Hedin [17]). The proposed approximation to the vertex gives $Z^{(\mathrm{QP})}=0.628$, which thus remains rather close to the QMC results 0.64 to 0.69 (at $r_{s}=3.99 a_{B}$ ) [6]. At the Fermi momentum $Z^{(\mathrm{QP})}$ can also be deduced from the discontinuity of the momentum distribution function $n_{k}$ [75-77]. In Fig. 4(e) we show $n_{k}$ as obtained by a straightforward integration of the smooth part of the spectral function $n_{k}=\int_{-\infty}^{\mu}(d \omega / 2 \pi) A(k, \omega)$ and by adding the singular contributions analytically. The $G^{(0)} W^{(0)}$ and vertex results are almost indistinguishable.

We finally analyze in Fig. 4(f) the quasiparticle lifetime, a measure of electronic correlations [78,79]. In ab initio calculations for realistic systems this quantity is typically estimated using the $G_{0} W_{0}$ approximation [80-82]. However, there have also been attempts to go beyond this level of theory by, e.g., including $T$-matrix diagrams. In Ref. [83] a reduction of the QP lifetime [an increase of $\Gamma$ by $50 \%$ (70\%) in relation to $G W$ for $\left.r_{s}=2.07(4.86)\right]$ has been predicted and explained by "the multiple scattering". Our findings show the opposite trend, i.e., an increase of the QP lifetime (reduction of $\Gamma$ by $-50 \%$ in relation to $G W$ for $\left.r_{s}=4\right]$.

Conclusions. - Numerous authors have emphasized that the inclusion of the vertex function should remedy the drawbacks of self-consistent calculations [25,27,30,84]. Using our recently proposed diagrammatic analysis we have been able to confirm these expectations and show that this is only a part of the whole picture. Additionally, other second-order processes appear. They can be best described in the language of scattering theory with the link provided by the PSD formalism $[51,52]$.

We fully characterized the spectral function of the 3D HEG in the $k-\omega$ plane. The main original features that we found are a second plasmon satellite for the holes, a redistribution of the spectral weight between the hole 
satellites, a reduction of the plasmon spectral weight for the particles, and a bandwidth reduction of the main QP band. So far these effects have only been partially captured by other, nondiagrammatic methods [58]. Our proposed approach has a universal character and can be extended to first-principle calculations of metals. In fact, in going from continuous to discrete translational symmetry the functions simply turn into matrix functions [e.g., $\Sigma(\mathbf{k}, \omega) \rightarrow$ $\Sigma_{G G^{\prime}}(\mathbf{k}, \omega)$ ], something that does not pose any conceptual difficulties for Monte Carlo momentum integration [85]. Alkali metals for which a vertex function has been partially included (typically using a model exchange-correlation kernel $[87,88]$ ) are a logical next step for our method. It is conceivable that higher order satellites can be accurately described by the combination of our approach with the cumulant expansion scheme outlined in Refs. [41,45]. A related approach based on the equation of motion coupledcluster theory has been put forward recently [89].

We acknowledge the CSC-IT Center for Science, Finland, for computational resources. Y. P. acknowledges support by the Deutsche Forschungsgemeinschaft (DFG) through Grant No. PA 1698/1-1. G. S. acknowledges funding by Ministero dell'Istruzione, dell'Università e della Ricerca (MIUR) Fondo per gli Investimenti della Ricerca di Base (FIRB) Grant No. RBFR12SW0J and European Commission (EC) funding through the Research and Innovation Staff Exchange (RISE) Co-ExAN (GA644076). R. v. L. would like to thank the Academy of Finland for support.

*yaroslav.pavlyukh@physik.uni-halle.de

[1] L. D. Landau and D. ter Haar, Collected Papers of L. D. Landau (Gordon and Breach, New York, 1967).

[2] P. Nozières and D. Pines, The Theory of Quantum Liquids, Advanced Book Classics (Westview Press, Perseus Books Group, Boulder, CO, 1999).

[3] G. Giuliani and G. Vignale, Quantum Theory of the Electron Liquid (Cambridge University Press, Cambridge, England, 2005).

[4] G. Stefanucci and R. van Leeuwen, Nonequilibrium ManyBody Theory of Quantum Systems: A Modern Introduction (Cambridge University Press, Cambridge, England, 2013).

[5] S. Moroni, D. M. Ceperley, and G. Senatore, Phys. Rev. Lett. 75, 689 (1995).

[6] M. Holzmann, B. Bernu, C. Pierleoni, J. McMinis, D. M. Ceperley, V. Olevano, and L. Delle Site, Phys. Rev. Lett. 107, 110402 (2011).

[7] E. Wigner, Phys. Rev. 46, 1002 (1934).

[8] A. W. Overhauser, Phys. Rev. Lett. 3, 414 (1959).

[9] G. Ortiz, M. Harris, and P. Ballone, Phys. Rev. Lett. 82, 5317 (1999).

[10] J. R. Trail, M. D. Towler, and R. J. Needs, Phys. Rev. B 68 , 045107 (2003).

[11] L. Baguet, F. Delyon, B. Bernu, and M. Holzmann, Phys. Rev. Lett. 111, 166402 (2013).

[12] S. Zhang and D. M. Ceperley, Phys. Rev. Lett. 100, 236404 (2008).
[13] P. Gori-Giorgi, F. Sacchetti, and G. B. Bachelet, Phys. Rev. B 61, 7353 (2000).

[14] P. Gori-Giorgi and J. P. Perdew, Phys. Rev. B 64, 155102 (2001).

[15] M. Petersilka, U. J. Gossmann, and E. K. U. Gross, Phys. Rev. Lett. 76, 1212 (1996).

[16] G. Onida, L. Reining, and A. Rubio, Rev. Mod. Phys. 74, 601 (2002).

[17] L. Hedin, Phys. Rev. 139, A796 (1965).

[18] C.-O. Almbladh, U. von Barth, and R. van Leeuwen, Int. J. Mod. Phys. B 13, 535 (1999).

[19] P. García-González and R. W. Godby, Phys. Rev. B 63, 075112 (2001).

[20] N. E. Dahlen and R. van Leeuwen, J. Chem. Phys. 122, 164102 (2005).

[21] A. Stan, N. E. Dahlen, and R. van Leeuwen, J. Chem. Phys. 130, 114105 (2009).

[22] B. I. Lundqvist, Phys. Kondens. Mater. 6, 193 (1967).

[23] B. I. Lundqvist, Phys. Kondens. Mater. 6, 206 (1967).

[24] B. I. Lundqvist, Phys. Kondens. Mater. 7, 117 (1968).

[25] B. Holm and U. von Barth, Phys. Rev. B 57, 2108 (1998).

[26] H. Yasuhara, S. Yoshinaga, and M. Higuchi, Phys. Rev. Lett. 83, 3250 (1999).

[27] Y. Takada, Phys. Rev. Lett. 87, 226402 (2001).

[28] N.-H. Kwong and M. Bonitz, Phys. Rev. Lett. 84, 1768 (2000).

[29] G. Pal, Y. Pavlyukh, H. C. Schneider, and W. Hübner, Eur. Phys. J. B 70, 483 (2009).

[30] G. D. Mahan and B. E. Sernelius, Phys. Rev. Lett. 62, 2718 (1989).

[31] G. Mahan, in Comments on Condensed Matter Physics, Comments on Modern Physics, Vol. 16 (Gordon and Breach Science Publishers, Lausanne, 1994), p. 333.

[32] P. A. Bobbert and W. van Haeringen, Phys. Rev. B 49, 10326 (1994).

[33] A. Schindlmayr and R. W. Godby, Phys. Rev. Lett. 80, 1702 (1998).

[34] P. Minnhagen, J. Phys. C 8, 1535 (1975).

[35] E. L. Shirley, Phys. Rev. B 54, 7758 (1996).

[36] Y. Takada and H. Yasuhara, Phys. Rev. Lett. 89, 216402 (2002).

[37] F. Bruneval, F. Sottile, V. Olevano, R. Del Sole, and L. Reining, Phys. Rev. Lett. 94, 186402 (2005).

[38] H. Maebashi and Y. Takada, Phys. Rev. B 84, 245134 (2011).

[39] D. C. Langreth, Phys. Rev. B 1, 471 (1970).

[40] B. Holm and F. Aryasetiawan, Phys. Rev. B 56, 12825 (1997).

[41] B. Gumhalter, Phys. Rev. B 72, 165406 (2005).

[42] J. J. Kas, J. J. Rehr, and L. Reining, Phys. Rev. B 90, 085112 (2014).

[43] M. Z. Mayers, M. S. Hybertsen, and D. R. Reichman, Phys. Rev. B 94, 081109 (2016).

[44] F. Caruso and F. Giustino, Eur. Phys. J. B 89, 238 (2016).

[45] B. Gumhalter, V. Kovač, F. Caruso, H. Lambert, and F. Giustino, Phys. Rev. B 94, 035103 (2016).

[46] For instance the cumulant expansion is exact for deep core states interacting with plasmons and leads to the spectrum with equally spaced satellites [39]. Yet, this assumption is less justified for the valence band excitations [47] overestimating the weight of higher order plasmon satellites 
(something that can be partially cured by taking multiple plasmon branches and their dispersion into account $[48,49])$.

[47] S. Doniach, Phys. Rev. B 2, 3898 (1970).

[48] M. Cini, J. Phys. C 19, 429 (1986).

[49] M. Guzzo, J. J. Kas, L. Sponza, C. Giorgetti, F. Sottile, D. Pierucci, M. G. Silly, F. Sirotti, J. J. Rehr, and L. Reining, Phys. Rev. B 89, 085425 (2014).

[50] P. Minnhagen, J. Phys. C 7, 3013 (1974).

[51] G. Stefanucci, Y. Pavlyukh, A.-M. Uimonen, and R. van Leeuwen, Phys. Rev. B 90, 115134 (2014); 93, 119906(E) (2016).

[52] A.-M. Uimonen, G. Stefanucci, Y. Pavlyukh, and R. van Leeuwen, Phys. Rev. B 91, 115104 (2015).

[53] D. Karlsson and R. van Leeuwen, Phys. Rev. B 94, 125124 (2016).

[54] We start with the zeroth approximation $\Delta \mu^{(0)}=$ $\operatorname{Re} \Sigma\left(k_{F}, 1 / 2 k_{F}^{2}\right)$ and perform two more calculations for $k=k_{F} \pm \Delta k$, where $\Delta k$ is a small number, typically a few percent of the Fermi momentum. The refined chemical potential shift is then given by $\Delta \mu=\Delta \mu^{(0)}+$ $\frac{1}{2}\left(\Delta \epsilon_{k_{F}+\Delta k}+\Delta \epsilon_{k_{F}-\Delta k}\right)$, where $\Delta \epsilon_{k}$ is the correlational shift.

[55] I.-W. Lyo and E. W. Plummer, Phys. Rev. Lett. 60, 1558 (1988).

[56] W. Ku, A. G. Eguiluz, and E. W. Plummer, Phys. Rev. Lett. 85, 2410 (2000).

[57] R. Maezono, M. D. Towler, Y. Lee, and R. J. Needs, Phys. Rev. B 68, 165103 (2003).

[58] See Supplemental Material at http://link.aps.org/ supplemental/10.1103/PhysRevLett.117.206402, which includes Refs. [59-67], for benchmarking and numerical details.

[59] C.-O. Almbladh, Phys. Scr. 32, 341 (1985).

[60] S. Engelsberg and J. R. Schrieffer, Phys. Rev. 131, 993 (1963).

[61] B. I. Lundqvist, Phys. Kondens. Mater. 9, 236 (1969).

[62] D. Vigil-Fowler, S. G. Louie, and J. Lischner, Phys. Rev. B 93, 235446 (2016).

[63] C. Biswas, A. K. Shukla, S. Banik, V. K. Ahire, and S. R. Barman, Phys. Rev. B 67, 165416 (2003).

[64] A. Bostwick, F. Speck, T. Seyller, K. Horn, M. Polini, R. Asgari, A. H. MacDonald, and E. Rotenberg, Science 328, 999 (2010).

[65] E. H. Hwang and S. Das Sarma, Phys. Rev. B 77, 081412 (2008).

[66] B. Roulet, J. Gavoret, and P. Nozières, Phys. Rev. 178, 1072 (1969).

[67] A. O. Gogolin, A. A. Nersesyan, and A. M. Tsvelik, Bosonization and Strongly Correlated Systems (Cambridge University Press, Cambridge, England, 1998).
[68] M. Polini, R. Asgari, G. Borghi, Y. Barlas, T. Pereg-Barnea, and A. H. MacDonald, Phys. Rev. B 77, 081411 (2008).

[69] If $W$ in $\Sigma_{a \bar{a}}$ is replaced by the bare Coulomb interaction the so-called second-order exchange SE is obtained. Its on-shell value is density independent and it is known analytically [70-72], $\Sigma_{2 x}\left(k_{F}, 1 / 2 k_{F}^{2}\right)=\left[2 \pi^{2} \ln (2) / 3-3 \zeta(3)\right] / 4 \pi^{2}$. This result represents a useful check for our numerical algorithms.

[70] L. Onsager, L. Mittag, and M. J. Stephen, Ann. Phys. 473, 71 (1966).

[71] P. Ziesche, Ann. Phys. 16, 45 (2007).

[72] M. L. Glasser and G. Lamb, J. Phys. A 40, 1215 (2007).

[73] Y. Pavlyukh, J. Berakdar, and A. Rubio, Phys. Rev. B 87, 125101 (2013).

[74] F. Aryasetiawan, L. Hedin, and K. Karlsson, Phys. Rev. Lett. 77, 2268 (1996).

[75] C. Mahaux and R. Sartor, Phys. Rep. 211, 53 (1992).

[76] P. Gori-Giorgi and P. Ziesche, Phys. Rev. B 66, 235116 (2002).

[77] V. Olevano, A. Titov, M. Ladisa, K. Hämäläinen, S. Huotari, and M. Holzmann, Phys. Rev. B 86, 195123 (2012).

[78] P. M. Echenique, J. M. Pitarke, E. V. Chulkov, and A. Rubio, Chem. Phys. 251, 1 (2000).

[79] Z. Qian and G. Vignale, Phys. Rev. B 71, 075112 (2005).

[80] V. P. Zhukov, F. Aryasetiawan, E. V. Chulkov, and P. M. Echenique, Phys. Rev. B 65, 115116 (2002).

[81] Y. Pavlyukh, J. Berakdar, and W. Hübner, Phys. Rev. Lett. 100, 116103 (2008).

[82] Y. Pavlyukh and J. Berakdar, J. Chem. Phys. 135, 201103 (2011).

[83] I. A. Nechaev and E. V. Chulkov, Phys. Rev. B 71, 115104 (2005).

[84] C. Verdozzi, R. W. Godby, and S. Holloway, Phys. Rev. Lett. 74, 2327 (1995).

[85] Because of the long-range character of the Coulomb interaction (the bare and to a lesser extent screened one) the integrations need to be extended beyond the boundaries of the first Brillouin zone. For $G W$ calculations the fast Fourier method has been proposed [86]. For higher dimensional integrals, such as in the present calculations, this approach becomes impractical, but can be remedied by the Monte Carlo integration featuring excellent scalability.

[86] H. N. Rojas, R. W. Godby, and R. J. Needs, Phys. Rev. Lett. 74, 1827 (1995).

[87] J. E. Northrup, M. S. Hybertsen, and S. G. Louie, Phys. Rev. Lett. 59, 819 (1987).

[88] J. Lischner, T. Bazhirov, A. H. MacDonald, M. L. Cohen, and S. G. Louie, Phys. Rev. B 89, 081108 (2014).

[89] J. McClain, J. Lischner, T. Watson, D. A. Matthews, E. Ronca, S. G. Louie, T. C. Berkelbach, and G. K.-L. Chan, Phys. Rev. B 93, 235139 (2016). 